\title{
Left hand dominance affects supra-second time processing
}

\author{
Carmelo Mario Vicario ${ }^{1}{ }^{*}$, Sonia Bonni ${ }^{2}$ and Giacomo Koch ${ }^{2}$ \\ Scuola Internazionale superiore di Studi Avanzati, Trieste, Italy \\ ${ }^{2}$ Istituto di Ricovero e Cura a Carattere Scientifico, Santa Lucia Foundation, Rome, Italy
}

\section{Edited by:}

Warren H. Meck, Duke University, USA

\section{Reviewed by:}

Martin Wiener, University of

Pennsylvania, USA

Deborah Lynn Harrington, VA San

Diego Healthcare System, USA

\section{*Correspondence:}

Carmelo Mario Vicario, Cognitive Neuroscience Sector, Scuola

Internazionale Superiore di Studi

Avanzati, via Bonomea 265, 34136

Trieste, Italy.

e-mail:cvicario@sissa.it;

Giacomo Koch, Istituto di Ricovero e

Cura a Carattere Scientifico, Santa

Lucia Foundation, via Ardeatina 306,

00179 Rome, Italy.

e-mail: g.koch@hsantalucia.it
Previous studies exploring specific brain functions of left- and right-handed subjects have shown variances in spatial and motor abilities that might be explained according to consistent structural and functional differences. Given the role of both spatial and motor information in the processing of temporal intervals, we designed a study aimed at investigating timing abilities in left-handed subjects. To this purpose both left- and right-handed subjects were asked to perform a time reproduction of sub-second vs. supra-second time intervals with their left and right hand. Our results show that during processing of the supra-second intervals left-handed participants sub-estimated the duration of the intervals, independently of the hand used to perform the task, while no differences were reported for the sub-second intervals. These results are discussed on the basis of recent findings on supra-second motor timing, as well as emerging evidence that suggests a linear representation of time with a left-to-right displacement.

\section{Keywords: left-handers, motor timing, time line, temporal accuracy}

\section{INTRODUCTION}

The literature exploring brain functions of left and right-handed subjects has shown variances in spatial and motor abilities that might be explained by consistent structural and functional differences.

In terms of visuo-spatial skills it is thought that in right-handed subjects, the right hemisphere has a prominent role in orienting attention toward the ipsi and contralateral space (Heilman et al., 1987). This leads to an over-representation of the left hemispace in comparison to the right hemispace (Kinsbourne, 1970). In lefthanded subjects, instead, this spatial unbalance seems to be almost absent (Sampaio and Chokron, 1992; Luh, 1995). A possible explanation for this difference may be obtained from the hemispheric activation model (Bradshaw et al., 1986; McCourt et al., 2001; Failla et al., 2003), which proposes that left-handed subjects have more equitably distributed visuo-spatial functions across cerebral hemispheres compared to right-handed subjects (McGlone and Davidson, 1973; Burnett et al., 1982; Vogel et al., 2003).

Nevertheless, the behavioral studies supporting this theoretical framework are rather contrasting. In the important study of Luh (1995) researchers reported a larger leftward bias in left-handed subjects than in right-handed subjects. However, the participants were only required to use their dominant hand to give the response (the right hand for right-handed subjects vs. the left hand for left-handed subjects). Similarly, Scarisbrick et al. (1987) provided evidence for an over-representation of the left hemispace in left-handed people. These authors asked right- and left-handed subjects to perform a visual line bisection task with each hand. The data reported that left-handed subjects using their left hand deviated significantly further left than right-handed subjects using their left hand.
Other interesting results were found in studies with children. Van Vugt et al. (2000) asked two groups of children (left-handed vs. right-handed) to bisect lines presented on the left, on the right or in the central position of a computer screen. According to the result reported by Scarisbrick et al. (1987) and Luh (1995), left-handed children showed a leftward bisection error in all hemispaces, while right-handed children displayed a left bias in the left hemispace, a right bias in the right hemispace, and no bias when the lines were presented in the center. On the other hand, there are at least two studies reporting different results. In the work of both Bradshaw et al. (1987) and Dellatolas et al. (1996) it was shown that left-handed subjects bisected horizontal lines toward the left of the objective midpoint when using the left hand, and more toward the right when using their right hand. A reversed pseudoneglect (rightward bias) was also more recently found in adult left-handed participants. Brodie and Dunn (2005) reported that left-handed participants only displayed a reversed pseudoneglect when using their preferred hand (left hand) and adopting a scan direction from right to left. Furthermore, a recent study of Begliomini et al. (2008) provides indirect evidence for a visuospatial function difference between these groups by reporting a different activity corresponding to the intraparietal sulcus (IPS), which represents a key area when attention as well as spatial updating are involved (Dehaene et al., 2003). In particular, a higher bilateral IPS activity was evident for right-handers compared to left-handers when using the right hand during a visuo-grasping task.

These anatomo-functional findings suggest that left- and righthanded people differ in their visuo-spatial functions, although several factors such as manual response, the allocation of visual attention (scan direction), and even the type of task performed by 
participants can critically affect the neural pattern and its' related behavioral outcome.

Differences between left and right-handers also emerge from studies that compare the motor cortical organization of these two groups. In a seminal study (Kimura, 1993) 10 right-handed and five left-handed subjects were instructed to make repetitive opposition movements of the thumb on each of the remaining four fingers when studied with functional magnetic resonance imaging (fMRI). Results showed a hemispheric asymmetry in the functional activation of the motor cortex during contralateral and ipsilateral movements which was significantly higher in right-handed subjects.

Similarly, Solodkin et al. (2001) reported that left-handers activated larger volumes and a larger number of brain areas than right-handers, and showed significantly less brain lateralization during a sequential movement task. A lower lateralization index was furthermore reported when left-handed subjects were asked to perform unimanual/bimanual tool-use pantomimes (Vingerhoets et al., 2011).

On the other hand, Begliomini et al. (2008) showed an increased activity corresponding to the right premotor cortex (PMC) of left-handed individuals (compared to the right-handed) when performing a grasping task with their left hand.

All these findings demonstrate consistent visuo-spatial and motor functional differences when comparing right- and lefthanded individuals.

It has been proposed that time may be represented along a leftto-right oriented mental time line, by analogy with numbers and other magnitudes, and that spatial attention plays a role in constructing this representation (Vicario et al., 2009). Moreover there is a large number of neuroimaging studies documenting a PMC engagement during the execution of several timing tasks.

We designed a research protocol aimed at exploring whether spatial and motor specificities documented in left-handed subjects could affect their ability in detecting temporal intervals. Numerous studies have already shown that a temporal underestimation may follow a leftward manipulation of the spatial attention, and that vice-versa a temporal overestimation may follow a rightward manipulation of the spatial attention (Vicario et al., 2007, 2008, 2009, 2011. Frassinetti et al., 2009. Oliveri et al., 2009). On the basis of such findings, we predicted that left-handed subjects would show a greater underestimation than right-handed subjects, in accordance with studies documenting a larger leftward bias of this group during the execution of visuo-spatial tasks (Scarisbrick et al., 1987; Luh, 1995).

In order to investigate such processes, we submitted our participants to a motor timing task, according to the evidence reporting important differences in the cortical organization of motor and sensorimotor regions of left-handed and right-handed subjects (Solodkin et al., 2001; Begliomini et al., 2008). This agrees with the suggestion of some potential role of sensorimotor regions in motor timing tasks (Vicario et al., 2010, 2011; Bengtsson et al., 2005; Lewis and Miall, 2006; Jantzen et al., 2007; Wiener et al., 2010).

Hence, we used a task engaging explicit time processing activity (Coull and Nobre, 2008) in the form of a motor response, in which subjects were asked to represent the timed duration within a sustained motor act (time reproduction task).

\section{MATERIALS AND METHODS PARTICIPANTS}

Fourteen left-handed (five males, nine females; mean age $=26 \pm 3.3$ $\mathrm{SD}$ ) and fourteen right-handed (five males, nine females; mean age $=26 \pm 3.3 \mathrm{SD}$ ) subjects participated in the experiment. All participants reported normal or corrected-to-normal vision and no neurological abnormalities. They were selected according to the Standard Handedness Inventory (Briggs and Nebes, 1975). Participants responded to this scale by indicating whether they use their right, left, or either hand for 12 common actions. We calculated the score for each subject by assigning a number from one to five to each response of the handedness Inventory (left $=1$; usually left $=2$; no preference $=3$; usually right $=4$; always right $=5$ ). In order to maximize the effects of handedness on the temporal performance, we excluded ambidextrous participants and participants totalizing an inventory score $>1.2$ and $<4.8$ (left-handed participants: mean $=1.005 \pm 0.022 \mathrm{SD}$, min-max range $1-1.083$; right-handed participants: mean $=4.981 \pm 0.049 \mathrm{SD}$, $\min -\max$ range 4.83-5). All subjects gave their written informed consent prior to their inclusion in the study and were naive in relation to its purpose. Subjects were compensated with $10 €$ for their participation, and specific information concerning the study was provided only after the subject had terminated all experimental sessions.

\section{APPARATUS AND STIMULI}

We used E-prime 1.2 software to create the visual stimuli and conduct the experiment. We used a version of the time reproduction task previously used by other authors (Jones et al., 2004; Koch et al., 2007; Vicario et al., 2010). Subjects sat at a distance of $60 \mathrm{~cm}$ opposite the monitor configured to a refresh rate of $100 \mathrm{~Hz}$. Subjects were asked to fixate a black cross of $0.2^{\circ}$ in diameter, centrally located on the screen. After $500 \mathrm{~ms}$, the black circle (test stimulus; size: $0.8^{\circ} \times 0.1^{\circ}$ ) appeared in the same location of the fixating cross; after a specified period, it disappeared. Immediately after the black circle disappeared, subjects were instructed to reproduce the interval they had just perceived by keeping the space bar on the computer keyboard pressed down with the index finger of their dominant hand. During such time they fixated a white screen. When they had judged that the same amount of time had elapsed, they had to release the space bar. Each session consisted in two separate and consecutive blocks. Following a study design previously adopted by other authors (Jones et al., 2004), we chose to challenge subjects in the reproduction of five different time intervals within each of the two blocks, in order to minimize the chance of a learning process throughout the performance of the task. Therefore, one block consisted of 50 trials in which subjects estimated five sub-second intervals $(500,600,700,800,900 \mathrm{~ms})$ each of which was presented for 10 repetitions in a randomized order; while the other block consisted of 50 trials in which subjects estimated five supra-second intervals (1500, 1600, 1700, 1800, $1900 \mathrm{~ms}$ ) with again 10 repetitions of each presented in randomized order. The reference stimulus was presented immediately before the test stimulus in each of the 50 trials of each block, and in both blocks the inter-trial interval was $2000 \mathrm{~ms}$. The presentation order of the sub-second and the supra-second blocks 
was counterbalanced within subjects in each group. Each block also consisted of two counterbalanced sessions in which participants were separately asked to perform the task by using their left or their right hand. Therefore our participants performed a total of four sessions. The performance of each subject on this task was analyzed as the mean difference expressed in milliseconds between groups. We did not test longer intervals to limit the length of the session, maintain a constant level of attention in participants, and to discourage subjects from adopting counting procedures to help themselves during the perception or reproduction of time intervals. No feedback concerning the quality of their performance was given to the subjects during the inter-trial interval.

\section{DATA ANALYSIS}

The reproduction performance in the time reproduction task was analyzed using ANOVA for repeated measures, with BLOCK (sub-second vs. supra-second) as the between-subject factor, and GROUP (left-handed subjects vs. right-handed subjects), MANUAL RESPONSE (left hand vs. right hand), and INTERVALS (500-900 vs. 1500-1900 ms) as within-subject factors. Reaction times (RTs) trials that fell 2.5 SDs above or below each individual mean for each experimental condition were excluded as outlier trials (sub-second block: Left-handers 2.29\%; Right-handers 2.42\%; supra-second block: Left-handers 2.57\%; Right-handers 2.86\%). Two tailed $t$-test analysis showed any significant between groups difference (Sub-second block $t=-0.210, p=0.836$; Supra-second block $t=-0.353, p=0.729)$. The mean response times were fit with a linear regression $(y=a x+y 0)$, and the slope and intercept values obtained for both groups were compared. Finally, as a measure of variability, we used the coefficient of variation $(\mathrm{CV}$; $\mathrm{SD} /$ mean response time).

Post hoc comparisons were performed using the NewmanKeuls post hoc test, and for all statistical analyses, a $p$ value of $<0.05$ was considered to be significant. Data analysis was performed using Statistica software, version 8.0, StatSoft, Inc., Tulsa, USA.

\section{RESULTS}

The three-way ANOVA for repeated measures on the accuracy did not detect a significant main effect of GROUP on the time reproduction task $[F(1,13)=3.8, p=0.074]$. Likewise there was no effect of the main factor MANUAL RESPONSE $[F(1,13)=2.1, p=0.175]$ and interaction terms BLOCK $\times$ MANUAL RESPONSE $[F(2,26)=2.4$, $p=0.143]$, GROUP $\times$ MANUAL RESPONSE $[F(2,26)=1.0$, $p=0.337]$, GROUP $\times$ INTERVALS $[F(2,26)=0.1, p=0.996]$ MANUAL RESPONSE $\times$ INTERVALS $[F(2,26)=0.2, p=0.937]$, BLOCK $\times$ GROUP $\times$ MANUAL RESPONSE $[F(3,39)=0.6$, $p=0.451] \quad$ BLOCK $\times$ GROUP $\times$ INTERVALS $[F(3,39)=0.8$, $p=0.508]$, BLOCK $\times$ MANUAL RESPONSE $\times \operatorname{INTERVALS~}[F(3$, $39)=0.4, \quad p=0.773], \quad$ GROUP $\times$ MANUAL $\quad$ RESPONSE $\times$ INTERVALS $[F(3,39)=2.1, p=0.097]$, and BLOCK $\times$ GROUP $\times$ INTERVALS $\times$ MANUAL RESPONSE $[F(4,52)=1.5, p=0.227]$. There was, instead, a significant main effect of the withinsubject factor BLOCK $[F(1,13)=323.7, p<0.001]$, INTERVALS $[F(1,13)=129.1, \quad p<0.001]$, and the interaction terms BLOCK $\times$ INTERVALS $[F(2,26)=3.2, p=0.020]$ and GROUP $\times \operatorname{BLOCK}[F(2,26)=4.8, p=0.046]$. Post hoc tests showed that left-handed subjects underestimated temporal durations only for the supra-second range of intervals $(p=0.008)$ when compared to right-handed subjects; whereas the reproduction performance did not differ between the two groups for the sub-second range of intervals ( $p=0.982$; Figure 1$)$.

A further one-tailed $t$-test analysis was performed only for the supra-second block, in order to asses a possible role of the hand used to generate the temporal response.

\section{WITHIN-SUBJECT ANALYSIS}

Left-handed group: left hand $M=1308.2 \mathrm{~ms} \pm 52.70$ vs. right hand $M=1239.2 \mathrm{~ms} \pm 54.55 t=0.909, p=0.186$; right-handed group: left hand $M=1442.5 \mathrm{~ms} \pm 57.76$ vs. right hand $M=1427.4 \mathrm{~ms} \pm 80.13 ; t=0.152, p=0.440$.

\section{BETWEEN-SUBJECT ANALYSIS}

Left hand: left-handed $M=1308.2 \mathrm{~ms} \pm 52.70$ vs. righthanded $M=1442.5 \mathrm{~ms} \pm 57.76, \quad t=-1.71, \quad p=0.048 ;$ right hand: left-handed $M=1239.2 \mathrm{~ms} \pm 54.55$ vs. right-handed $M=1427.4 \mathrm{~ms} \pm 80.13, t=-1.94, p=0.032$.

The within-subject analysis did not show significant differences among the analyzed conditions, while the between-subject analysis showed that left-handers underestimated time with both their left and right hand.

Since we obtained a significant difference for the supra-second block, we tried to fit the accuracy for the supra-second intervals range with a linear regression model and looked for differences in slope or intercept (Figure 2).

The ANOVA performed on the slope scores did not detect a significant main effect of GROUP $[F(1,13)=0.8, p<0.373]$ and HAND $[F(1,13)=0.8, p<0.361]$ and the interaction term GROUP $\times$ BLOCK $[F(1,13)=2.5, p=0.137]$. Likewise the ANOVA performed on the intercept scores did not detect a significant main effect of GROUP $[F(1,13)=0.04, p<0.836]$ and HAND $[F(1,13)=1.84, p<0.198]$ and the interaction term GROUP $\times \operatorname{BLOCK}[F(1,13)=0.20, p=0.658]$.

Finally, the ANOVA for repeated measures on the CV scores for both groups did not detect a significant interaction for the factors BLOCK $\times$ GROUP $[F(1,13)=0.4$, $p=0.503], \quad$ BLOCK $\times$ GROUP $\times$ MANUAL RESPONSE $[F(1$, $13)=0.7, p=0.797]$. However we detected a significant main effect of GROUP $[F(1,13)=7.7, p=0.015]$, which indicated lower variability of the left-handers $(0.228 \pm 0.013)$ compared to the right-handers $(0.256 \pm 0.012)$. Likewise there was a main effect for the MANUAL RESPONSE $[F(1$, $13)=17.0, p<0.001]$, BLOCK $[F(1,13)=53.8, p<0.001]$, INTERVALS $[F(1,13)=63.0, p<0.001]$ factor, and the interaction effect BLOCK $\times$ MANUAL RESPONSE $[F(1,13)=42.7$, $p<0.001]$, GROUP $\times$ MANUAL RESPONSE $[F(1,13)=47.5$, $p<0.001]$, BLOCK $\times$ INTERVALS $[F(1,13)=30.7, p<0.001]$, GROUP $\times$ INTERVALS $[F(1,13)=25.7, p<0.001], \quad$ MANUAL RESPONSE $\times$ INTERVALS $[F(1,13)=4.3, p<0.004]$, and the interaction terms BLOCK $\times$ GROUP $\times$ INTERVALS $[F(1,13)=6.0, p<0.001]$, BLOCK $\times$ MANUAL RESPONSE $\times$ INTERVALS $[F(1,13)=7.7, p<0.001]$, GROUP $\times$ MANUAL 


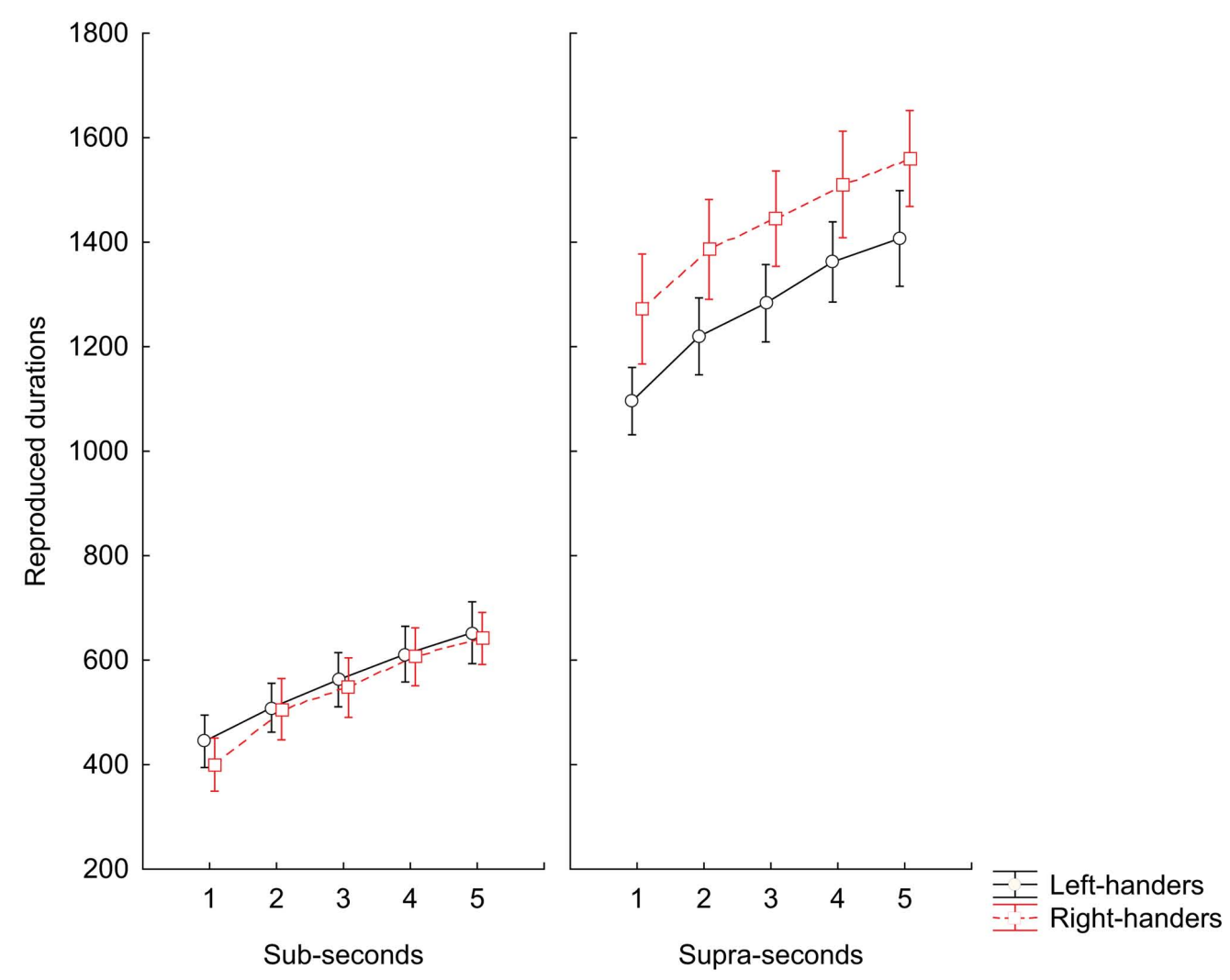

FIGURE 1 |Time reproduction task. Means of median RT values during reproduction of sub-second and supra-second intervals for left-handed subjects and right-handed subjects participants. The vertical bars indicate SE mean.

RESPONSE $\times$ INTERVALS $[F(1,13)=11.8, p<0.001]$, and BLOCK $\times$ GROUP $\times$ INTERVALS $\times$ MANUAL RESPONSE $[F(1$, 13) $=12.1, p=0.001]$.

Post hoc analysis on the interaction factors BLOCK $\times$ GROUP $\times$ INTERVALS $\times$ MANUAL RESPONSE, when using the left hand, reported that right-handers were more variable than left-handers in reproducing almost all sub-second intervals $(600 \mathrm{~ms}, p=0.003$; $700 \mathrm{~ms}, p=0.004 ; 800 \mathrm{~ms}, p<0.001)$; however the accuracy pattern was inverted at $900 \mathrm{~ms}$ in which case left-handers were more variable than right-handers $(p<0.001)$; any difference was reported when participants reproduced intervals at $500 \mathrm{~ms}$.

We observed that right-handers were also more variable than left-handers in reproducing $1500 \mathrm{~ms}(p<0.001)$, but more accurate at $1700 \mathrm{~ms}(p<0.003)$ and at $1900 \mathrm{~ms}(p<0.001)$. Any significant differences was reported for 1600 and $1800 \mathrm{~ms}$. (see Figure 3 for further details).

A similar pattern of higher variability of the right-handers was reported when using their right hand in reproducing subsecond intervals such as $500 \mathrm{~ms}(p<0.001) ; 600 \mathrm{~ms}(p<0.001)$, and $700 \mathrm{~ms}(p<0.001)$. Any difference was reported in reproducing 800 and $900 \mathrm{~ms}$. Our right-handers were more variable than left-handers even when asked to reproduce supra-second intervals (1500 ms, $p<0.001 ; 1600 \mathrm{~ms}, p<0.001 ; 1700 \mathrm{~ms}, p<0.001$; $1800 \mathrm{~ms}, p<0.001)$. However, any difference was reported at $1900 \mathrm{~ms}$ (see Figure 4 for further details).

\section{DISCUSSION}

The aim of the present work was to assess the ability of left-handed subjects to reproduce temporal intervals within the sub-second and the supra-second duration ranges. The rationale of our study was based on previous findings that reported substantial differences in the processing of visuo-spatial and motor information between left-handed and right-handed groups (Scarisbrick et al., 1987; Peters, 1991; Sampaio and Chokron, 1992; Luh, 1995; Rushworth et al., 1997; Solodkin et al., 2001; Begliomini et al., 2008; Vingerhoets et al., 2011). During processing of the supra-second intervals left-handed participants sub-estimated the duration of the intervals, independently of the hand used to perform the task, while no differences were reported for the sub-second intervals. The CV analysis showed that the performances of left-handed participants were significantly less variable than right-handed participants. This result was particularly evident for the timing of sub-second intervals, though we did not detect a significant difference between groups when comparing the raw data of this temporal range. Left-handed subjects were also less variable in the timing of supra-second intervals when using their right hand to generate the temporal response.

On the other hand, for the timing of supra-second intervals the parameters of variability were much more flexible between groups when subjects were asked to use their left hand to reproduce the temporal intervals. This result may imply that the hemispheric 


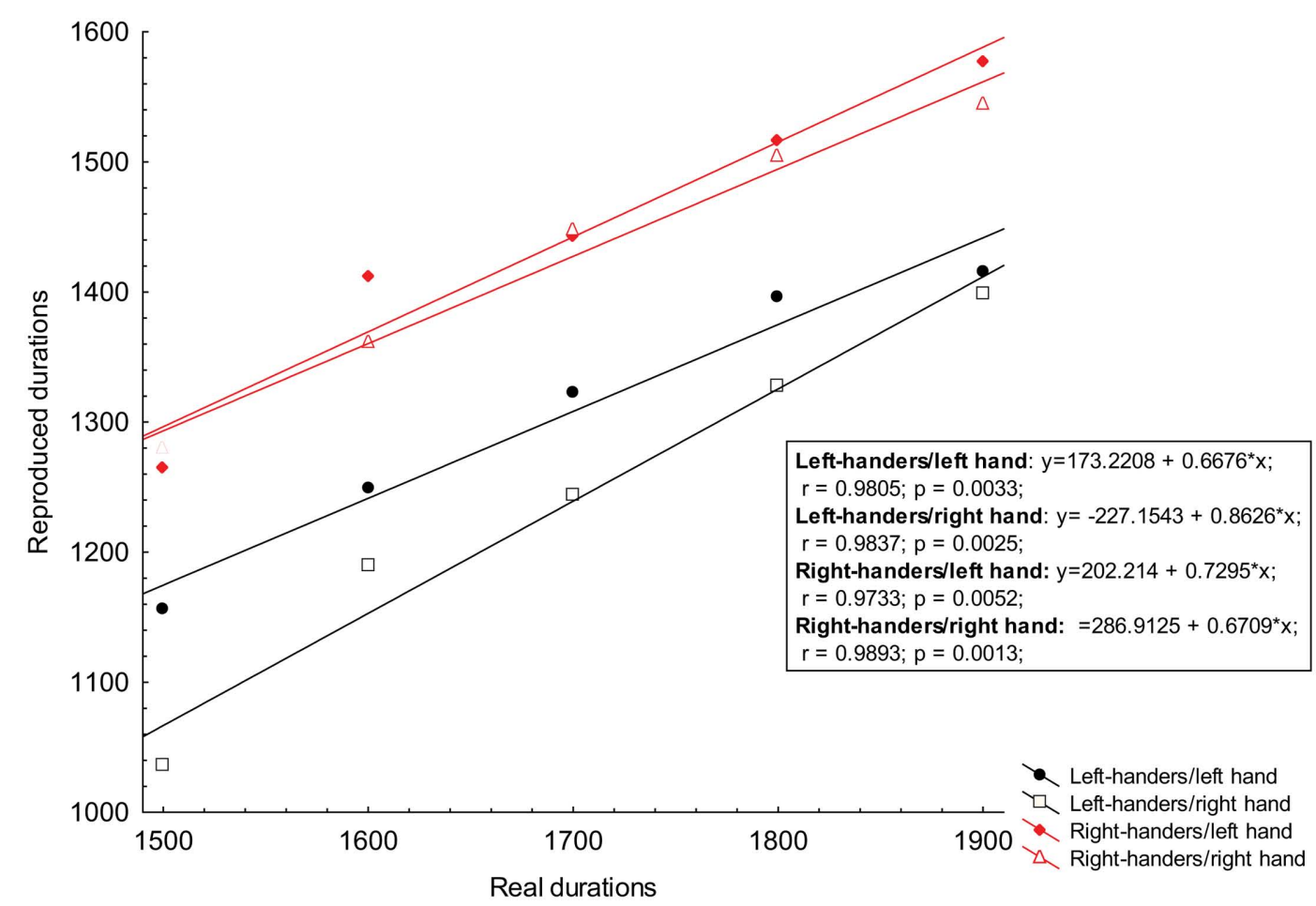

FIGURE 2 |The obtained reproduction scores for supra-second intervals were plotted against references durations and then fit with a linear regression. The ordinate represents the reproduced time for both left-handers and right-handers groups performing the task with their left and right hand; The abscissa represents the real durations of reference stimuli. Both scores are expressed in milliseconds. Equations derived from each linear regression, correlation, and $R$ square for each condition are displayed in the graph. activation associated to the limb used for generating the response has different effects on the timing variability itself.

The present findings could be related to the different underlying neural circuits that are thought to be involved in the processing of sub-second and supra-second time intervals, although there are several common neural activation mechanisms involved in both temporal domains (Koch et al., 2009; Wiener et al., 2010, 2011). In particular, when subjects are required to quickly estimate the passage of sub-second time intervals or perform sub-second motor timing tasks, and when time is computed in relation to precise salient events, it is thought that the neural activity of the cerebellum and the superior temporal gyrus is most crucial. Conversely, the parieto-frontal circuits mainly in the right hemisphere seem to be the most implicated processing of supra-second time intervals (included motor timing tasks), and when time is processed in conjunction with other cognitive functions (Koch et al., 2009).

In the group of left-handed subjects the time reproduction of supra-second intervals was biased toward a sub-estimation pattern. On the other hand, left-handed subjects did not differ from controls (right-handed subjects) in the reproduction of sub-second intervals. Data from the CV analysis suggest that the variability cannot be responsible of the current sub-estimation pattern found in the left-handed participants. In fact, although the sub-estimation pattern founded in our left-handed participants can be explained in terms of a minor temporal precision (given the greater temporal gap from the real durations), the lower variability found in the performance of left-handers indicates a potential difference in the clock speed between these two groups for the timing of supra-second durations. This view is furthermore corroborated by the absence of a difference in accuracy when reproducing sub-second intervals in presence of a significant difference in the CV.

A prediction deriving from the hemispheric activation model is that the selective activation of one hemisphere by a unimanual response could result in an enhancement of the spatial representation contralateral to the cerebral hemisphere activated. Thus, in accordance with previous studies on left-handed subjects, (e.g., Bradshaw et al., 1987) who showed a leftward bias when bisecting a line with their left hand and a rightward bias when bisecting a line with their right hand, it is reasonably likely to expect a similar bias on temporal performance, depending of the hand used for responding. However, the GROUP $\times$ MANUAL RESPONSE factor was not significant. Since we did not test visuo-spatial skills in our participants, we cannot exclude a leftward bias dominance in our left-handed participants independently from the hand used for generating the temporal response. This suggestion is confirmed by the between-subject $t$-test analysis for accuracy reported in the current study.

Therefore the attentional orientation bias of the right hemisphere toward the left hemispace seems to be accentuated in left-handed subjects in relation to the frequency of use of their 


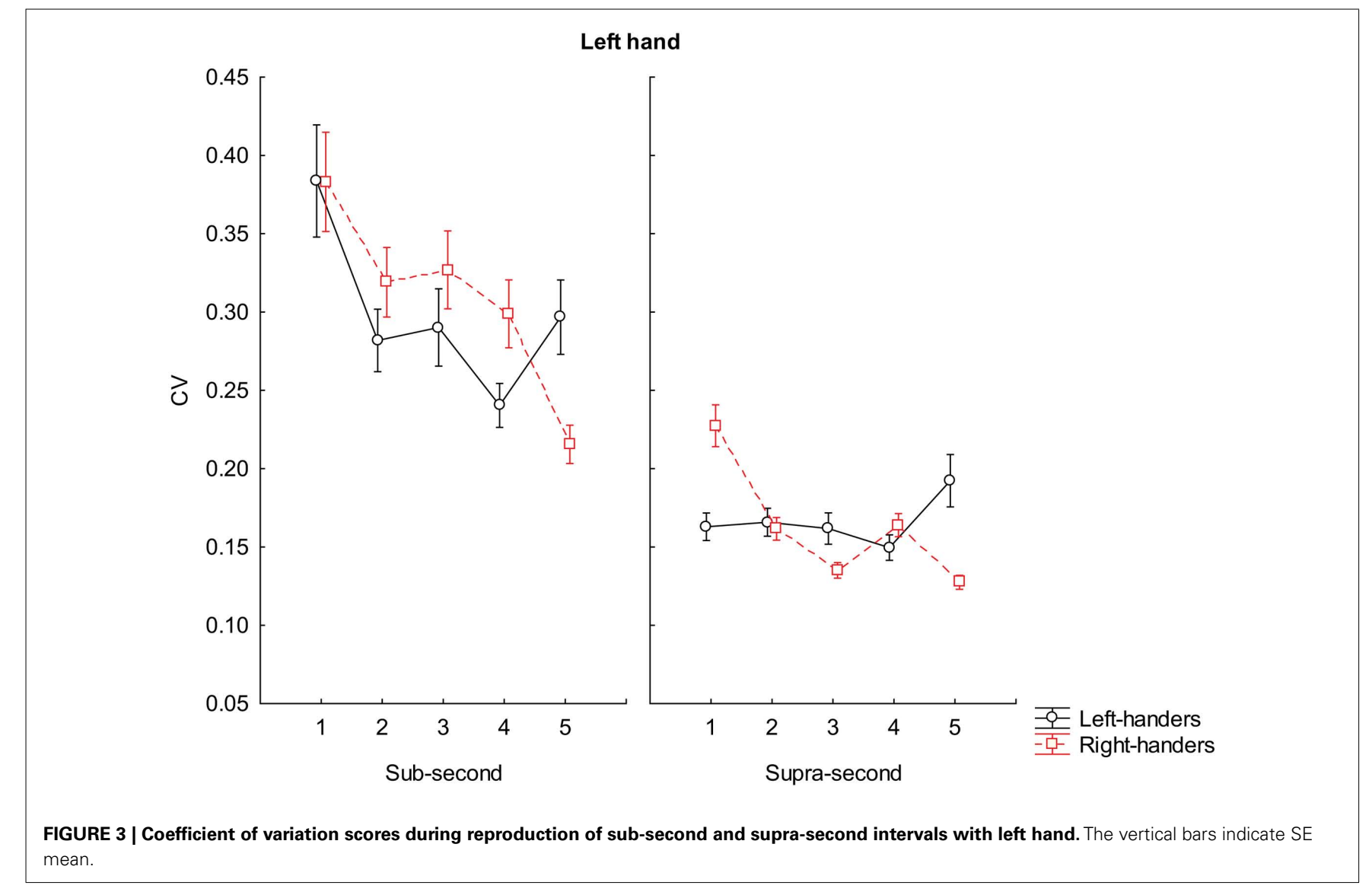

left hand in their everyday activities. This could, in turn, account for the current temporal underestimation.

Indirect evidence supporting the possible impact of the attentional orientation bias of the right hemisphere toward the left hemispace on time originates from an important study by Polzella et al. (1977). The authors of the study found that the mean judged duration of patterns flashed to the left visual field was significantly less than the mean judged duration of patterns flashed to the right visual field.

In a recent study Vicario et al. (2007) tested the effects of left vs. rightward optokinetic stimulation on time comparison tasks of stimuli presented in a central position. The main results showed that directing attention toward the right hemispace induces time overestimation, while directing it toward the left hemispace time underestimation compared to baseline (Oliveri et al., 2009). In this setting, we might hypothesize that the spatial attention leftward bias reported in the studies exploring the performance of left-handed subjects in a line bisection task (Scarisbrick et al., 1987; Luh, 1995; Van Vugt et al., 2000), is responsible for the above-mentioned temporal sub-estimation.

An alternative - not mutually exclusive - explanation for the result we observe could be given by the functional and/or structural differences in motor and sensorimotor regions between the two groups we tested. Motor timing performance seems to depend on the degree of co-activation between the basal ganglia and the supplementary motor area (SMA), the dorsolateral prefrontal cortex, and the cerebellum (see Witt et al., 2008 for a meta analysis). Together with other brain regions, the right SMA activity seems to be particularly related to the processing of supra-second motor timing tasks (Wiener et al., 2010). A possibility is that the current timing pattern would be related to the functional specificities concerning the SMA of left-handed subjects, as reported by Solodkin et al. (2001). In the context of this discussion, a different explanation could be referred to the functional differences across other regions involved in the motor control. For example, there is a large number of neuroimaging studies documenting the engagement of PMC during the execution of several timing tasks. It was recently shown (Begliomini et al., 2008) that the right PMC activity is increased in left-handed individuals (compared to the right-handed) who were asked to perform a grasping task.

The superiority of right-handed subjects in motor tasks such as finger-tapping, which is likely related to the superiority in the neural processing of their left hemisphere, emerges from at least two neuropsychological studies (Peters, 1991; Rushworth et al., 1997); thus the sub-estimation bias of our left-handed group could be interpreted in terms of a lower accuracy as a consequence of the lower motor control of this group.

The language dominance hemisphere view is also in agreement with the results of the first report of lateralized differences on perceptual timing (Efron, 1963). In his study, the author showed that for right-handers, the sensation of subjective simultaneity 


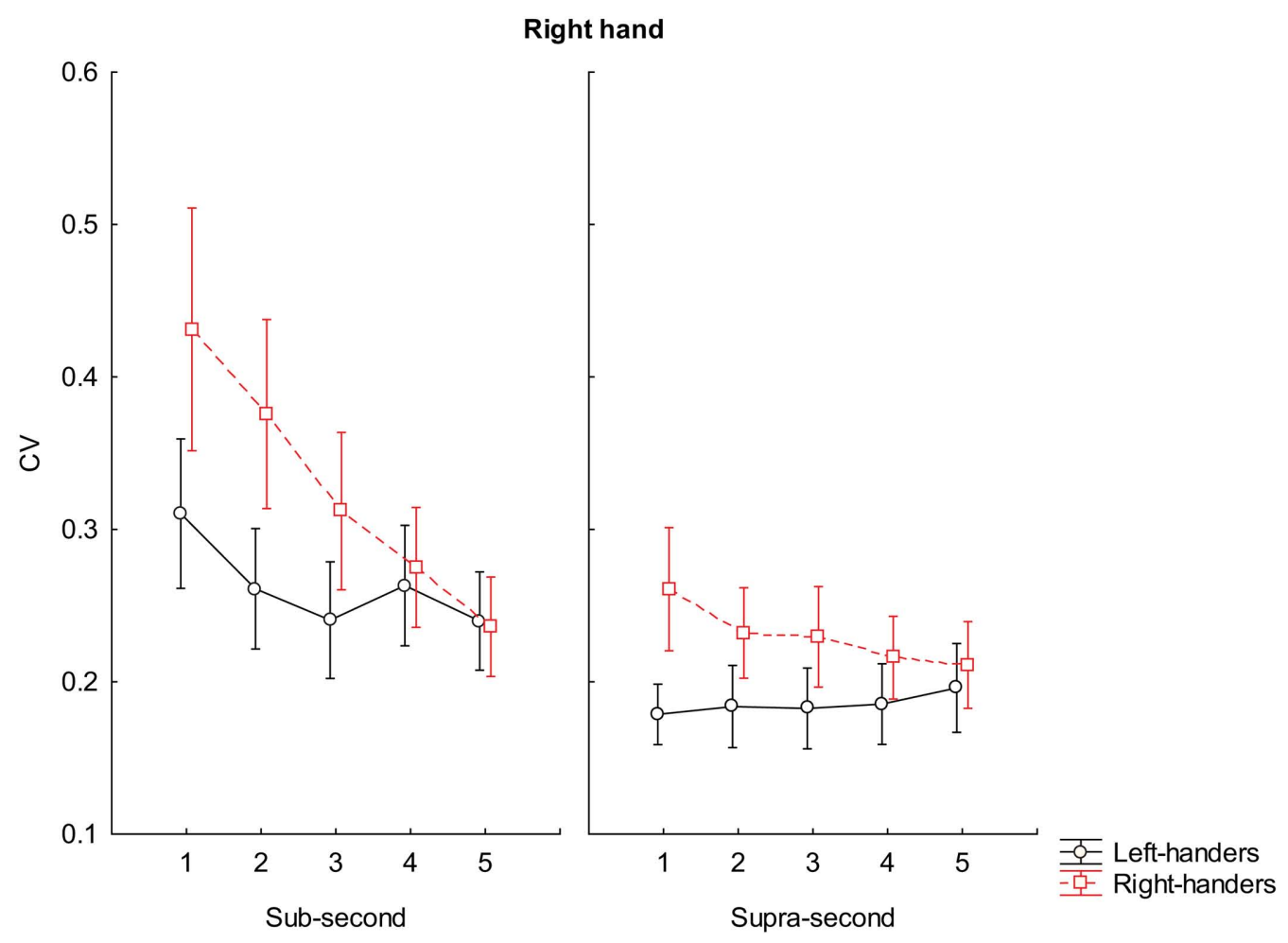

FIGURE 4 | Coefficient of variation scores during reproduction of sub-second and supra-second intervals with right hand. The vertical bars indicate SE mean.

of a pair of lateralized stimuli (brief light flashes and cutaneous shocks were used) was felt when the stimulus delivered to the left visual field or left index finger preceded the stimulus delivered to the right side by 3-4 ms. Instead, the point of subjective simultaneity for a group of left-handers did not differ from zero.

Although the hemispheric difference reported above can differentially impact the current results, other factors, such as scanning strategies and the manual response may influence the final timing outcome.

\section{REFERENCES}

Begliomini, C., Nelini, C., Caria, A., Grodd, W., and Castiello, U. (2008). Cortical activations in humans grasp-related areas depend on hand used and handedness. PLoS ONE 3, e3388. doi:10.1371/journal.pone. 0003388

Bengtsson, S. L., Ehrsson, H. H., Forssberg, H., and Ullen, F. (2005). Effector independent voluntary timing: behavioural and neuroimaging evidence. Eur. J. Neurosci. 22, 3255-3265.

Bradshaw, J. L., Bradshaw, J. A., Nathan, G., Nettleton, N. C., and Wilson, L. E. (1986). Leftwards error in bisecting the gap between two points: stimulus quality and hand effects. Neuropsychologia 24, 849-855.

Bradshaw, J. L., Nettleton, N. C. Wilson, L. E., and Bradshaw, C. S. (1987). Line bisection by lefthanded preschoolers: a phenomenon of symmetrical neglect. Brain Cogn. 6, 377-385.

Briggs, G. G., and Nebes, R. D. (1975). Patterns of hand preference in a student population. Cortex 11, 230-238.

Brodie, E. E., and Dunn, E. M. (2005). Visual line bisection in sinistrals and dextrals as a function of hemispace, hand, and scan direction. Brain Cogn. 58, 149-156.

Burnett, S. A., Lane, D. M., and Dratt, L. M. (1982). Spatial ability

All these studies could be useful in explaining the alteration of the temporal performance in left-handed subjects as well as the direction of the bias toward sub-estimation.

To the best of our knowledge, our study is the first to explore and report differences in explicit timing tasks between left- and right-handed subjects. However, our study presents an important limitation, given our subjects performed only a time reproduction task, which do not allow an investigation of the perceptual domain that is pertinent of a different timing paradigms such as a time discrimination task.

and handedness. Intelligence 6 , 57-68.

Coull, J. T., and Nobre, A. C. (2008). Dissociating explicit timing from temporal expectation with fMRI. Curr. Opin. Neurobiol. 18 137-144.

Dehaene, S., Piazza, M., Pinel, P., and Cohen, L. (2003). Three parietal circuits for number processing. Cogn. Neuropsychol. 20, 487-506.

Dellatolas, G., Coutin, T., and Agostini, M. (1996). Bisection and perception of horizontal lines in normal children. Cortex 32, 705-715.

Efron, R. (1963). Temporal perception, Aphasia and D'e J'a vu. Brain 86, 403-424.
Failla, C. V., Sheppard, D. M., and Bradshaw, J. L. (2003). Age and responding-hand related changes in performance of neurologically normal subjects on the line-bisection and chimeric-faces tasks. Brain Cogn. 52, 353-363.

Frassinetti, F., Magnani, B., and Oliveri, M. (2009). Prismatic lenses shift time perception. Psychol. Sci. 20, 949-954.

Heilman, K. M., Bowers, D., Valenstein, E., and Watson, R. T. (1987). "Hemispace and hemispatial neglect," in Neurophysiological and Neuropsychologial Aspects of Spatial Neglect, ed. M. Jeannerod (Amsterdam: Elsevier), 115-150. 
Jantzen, K. J., Oullier, O., Marshall, M., Steinberg, F. L., and Kelso, J. A. (2007). A parametric fMRI investigation of context effects in sensorimotor timing and coordination. Neuropsychologia 45, 673-684.

Jones, C. R., Rosenkranz, K., Rothwell, J. C., and Jahanshahi, M. (2004). The right dorsolateral prefrontal cortex is essential in time reproduction: an investigation with repetitive transcranial magnetic stimulation. Exp. Brain Res. 158, 366-372.

Kimura, D. (1993). Neuromotor Mechanisms in Human Communication. New York: Oxford University Press.

Kinsbourne, M. (1970). The cerebral basis of lateral asymmetries in attention. Acta Psychol. 33, 193-201.

Koch, G., Oliveri, M., and Caltagirone, C. (2009). Neural networks engaged in milliseconds and seconds time processing: evidence from transcranial magnetic stimulation and patients with cortical or subcortical dysfunction. Philos. Trans. R. Soc. Lond. B Biol. Sci. 364, 1907-1918. [Review].

Koch, G., Oliveri, M., Torriero, S., Salerno, S., Lo Gerfo, E., and Caltagirone, C. (2007). Repetitive TMS of cerebellum interferes with millisecond time processing. Exp. Brain Res. 179, 291-299.

Lewis, P. A., and Miall, R. C. (2006). Remembering the time: a continuous clock. Trends Cogn. Sci. (Regul. Ed.) 10, 401-406.

Luh, K. E. (1995). Line bisection and perceptual asymmetries in normal individuals: what you see is not what you get. Neuropsychology 9, 435-448.
McCourt, M. E., Freeman, P., Tahmahkera-Stevens, C., and Chaussee, M. (2001). The influence of unimanual response on pseudoneglect magnitude. Brain Cogn. 45, 52-63.

McGlone, J., and Davidson, W. (1973). The relation between cerebral speech laterality and spatial ability with special reference to sex and hand preference. Neuropsychologia 11, 105-113.

Oliveri, M., Koch, G., Salerno, S., Torriero, S., Lo Gerfo, E., and Caltagirone, C. (2009). Representation of time intervals in the right posterior parietal cortex: implications for a mental time line. Neuroimage 46, 1173-1179.

Peters, M. (1991). Laterality and motor control. Ciba Found. Symp. 162, 300-308.

Polzella, D. J., daPolito, F., and Hinsman, C. (1977). Cerebral asymmetry in time perception. Percept. Psychophys. 21, 187-192.

Rushworth, M. F., Nixon, P. D., Renowden, S., Wade, D. T., and Passingham, R. E. (1997). The left parietal cortex and motor attention. Neuropsychologia 35, 1261-1273.

Sampaio, E., and Chokron, S. (1992). Pseudoneglect and reversed pseudoneglect among left-handers and right-handers. Neuropsychologia 30, 797-805.

Scarisbrick, D. J., Tweedy, J. R., and Kuslansky, G. (1987). Hand preference and performance effects on line bisection. Neuropsychologia 25, 695-699.

Solodkin, A., Hlustik, P., Noll, D. C., and Small, S. L. (2001). Lateralization of motor circuits and handedness during finger movements. Eur. J. Neurol. 8, 425-434.

Van Vugt, P., Fransen, I., Creten, W. and Paquier, P. (2000). Line bisection performances in 650 normal children. Neuropsychologia 35, 703-715.

Vicario, C. M., Caltagirone, C., and Oliveri, M. (2007). Optokinetic stimulation affects temporal estimation in healthy humans. Brain Cogn. 64 68-73.

Vicario, C. M., Martino, D., Pavone, E. F. and Fuggetta, G. (2011). Lateral head turning affects temporal memory. Percept. Mot. Skills 113, 3-10.

Vicario, C. M., Martino, D., Spata, F., Defazio, G., Giacchè, R., Martino, V., Rappo, G., Pepi, A. M., Silvestri, P. R., and Cardona, F. (2010). Time processing in children with Tourette's syndrome. Brain Cogn. 73, 28-34.

Vicario, C. M., Pecoraro, P., Turriziani, P., Koch, G., Caltagirone, G., and Oliveri, M. (2008). Relativistic compression and expansion of experiential time in the left and right space. PLoS ONE 3, e1716. doi:10.1371/journal.pone.0001716

Vicario, C. M., Rappo, G., Pepi, A., and Oliveri, M. (2009). Timing flickers across sensory modalities. Perception 38, 1144-1151.

Vingerhoets, G., Acke, F., Alderweireldt, A. S., Nys, J., Vandemaele, P. and Achten, E. (2011). Cerebral lateralization of praxis in rightand left-handedness: same pattern, different strength. Hum. Brain Mapp. doi:10.1002/hbm.21247. [Epub ahead of print].

Vogel, J. L., Bowers, C. A., and Vogel, D. S. (2003). Cerebral lateralization of spatial abilities: a meta-analysis. Brain Cogn. 52, 197-204.

Wiener, M., Lohoff, F. W., and Coslett, H. B. (2011). Double dissociation of dopamine genes and timing in humans. J. Cogn. Neurosci. 23, 2811-2821.

Wiener, M., Turkeltaub, P., and Coslett, H. B. (2010). The image of time: a voxel-wise meta-analysis. Neuroimage 49, 1728-1740.

Witt, S. T., Laird, A. R., and Meyerand, M. E. (2008). Functional neuroimaging correlates of finger-tapping task variations: an ALE meta-analysis. Neuroimage 42, 343-356.

Conflict of Interest Statement: The authors declare that the research was conducted in the absence of any commercial or financial relationships that could be construed as a potential conflict of interest.

Received: 18 July 2011; paper pending published: 29 July 2011; accepted: 30 September 2011; published online: 24 October 2011.

Citation: Vicario CM, Bonní $S$ and Koch $G$ (2011) Left hand dominance affects supra-second time processing. Front. Integr. Neurosci. 5:65. doi: 10.3389/fnint.2011.00065

Copyright (C) 2011 Vicario, Bonní and Koch. This is an open-access article subject to a non-exclusive license between the authors and Frontiers Media SA, which permits use, distribution and reproduction in other forums, provided the original authors and source are credited and other Frontiers conditions are complied with. 